\title{
Effects of Vildagliptin/Metformin Therapy on Patient- Reported Outcomes: Work Productivity, Patient Satisfaction, and Resource Utilization
}

\author{
Stefano Genovese · Donatella Tedeschi
}

To view enhanced content go to www.advancesintherapy.com Received: November 5, 2012 / Published online: February 20, 2013

(c) The Author(s) 2013. This article is published with open access at Springerlink.com

\section{ABSTRACT}

Introduction: Type 2 diabetes mellitus (T2DM) is associated not only with high direct healthcare costs, but also with indirect costs, as diabetic complications and the disease itself result in loss of productivity. Vildagliptin is a novel dipeptidyl peptidase-4 inhibitor that is given either alone or in combination with oral hypoglycemic drugs, including metformin. The study was designed to assess the hypothesis that fixed-combination vildagliptin/metformin improves work productivity measured as Work Productivity and

\section{S. Genovese* $(\square)$}

Cardiovascular and Metabolic Department, IRCCS

Multimedica, Via Milanese, 300, 20099, Sesto San

Giovanni (Milano), Italy

e-mail: stefano.genovese@multimedica.it

D. Tedeschi

Medical Department, Novartis Farma S.p.A, Largo

Umberto Boccioni, 1, 21040 Origgio (Varese), Italy

${ }^{*}$ On behalf of the Investigator's team

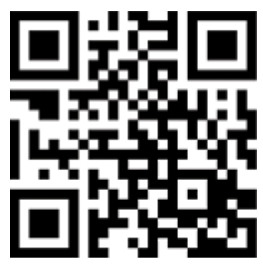

Enhanced content for Advances in Therapy articles is available on the journal web site: www.advancesintherapy.com
Activity Impairment (WPAI) scores. Secondary objectives were the assessment of patient satisfaction by means of the Diabetes Treatment Satisfaction Questionnaire (DTSQs), the change in anthropometric measurements and in glucose control (glycated hemoglobin [HbA1c]), and the evaluation of resource utilization (Resources Utilization Questionnaire).

Methods: This study was an addendum to a mandatory, prospective, observational study carried out by the Italian Medicines Agency (Agenzia Italiana del Farmaco [AIFA]) in 49 diabetes centers in Italy. The addendum included 1,046 adult outpatients with a diagnosis of T2DM, who were no longer responding to metformin monotherapy. Patients were observed for up to 1 year.

Results: Mean activity impairment improved by $40.6 \%$ (15.4 \pm 17.4 vs. $26.1 \pm 24.4 ; P<0.0001)$, absenteeism by $49.9 \%(2.0 \pm 9.4$ vs. $3.8 \pm 13.3$; $P=0.0076)$, and total work productivity by $37.6 \%$ $(14.9 \pm 15.9$ vs. $21.5 \pm 24.6 ; P<0.0001)$. This resulted in a reduction of the annual indirect cost due to impaired productivity of $€ 400$ per working patient and $€ 135$ per patient in general. The DTSQ score increased by 30.2\% (29.6 \pm 5.6 vs. $22.8 \pm 6.9 ; P<0.0001)$. The satisfaction rate increased from baseline by $44.7 \%$; the hyperglycemia-free or almost hyperglycemia-free 
perception rate by $37.9 \%$; and the hypoglycemiafree or almost hypoglycemia-free rate by $15.2 \%$. Mean healthcare costs per patients diminished by $19.2 \%$ in the second semester of treatment.

Conclusion: This observational study suggests that the fixed combination of vildagliptin/ metformin increases work productivity, reducing indirect costs, and improves quality of life, especially in terms of perception of blood glucose variability, in patients with T2DM.

Keywords: Fixed combination; Healthcare costs; Metformin; Patient-reported outcomes; Productivity; Resources; Type 2 diabetes mellitus; Vildagliptin

\section{INTRODUCTION}

European and US studies have shown that patients with type 2 diabetes mellitus (T2DM) account for $3-5 \%$ of the total population, but consume up to $15-20 \%$ of total healthcare resources [1]. According to the European CODE-2 (Costs of Diabetes in Europe-Type 2) study [2], the reason for this is frequent hospitalizations due to diabetic complications, which account for more than half of the healthcare costs (55\%) due to diabetes. The situation is alarming for healthcare budgets as the worldwide prevalence of T2DM is continually increasing: the World Health Organization (WHO) has estimated that it amounted to 171 million patients in 2000 and will more than double over 30 years, reaching 366 million in 2030 [3].

Diabetes is associated also with indirect costs, as diabetic complications reduce the patient's ability to work, resulting in loss of productivity and need for social service support. The Centers for Disease Control in the US have estimated that patients with diabetes lose on average 8.3 days of work per year versus 1.7 days per year for patients without diabetes [4], and a European study showed that both patients with T2DM and their carers lose income, especially when the patients experience diabetic complications [5]. According to the CODE-2 study, overall indirect costs of absenteeism related to diabetes in Italy amount to $€ 234$ million [6].

According to international guidelines, the management of T2DM includes the implementation of a healthy lifestyle, the introduction of metformin, which has proved to be able to reduce the risk of diabetic complications and death, and the use of other pharmacological options, as most patients require the addition of a second oral antidiabetic drug [7-9].

Vildagliptin is a dipeptidyl peptidase-4 (DPP-4) inhibitor that has proved to be effective in reducing glycated hemoglobin (HbA1c) without weight gain and with minimal hypoglycemia, when it is given as monotherapy or in combination with the most commonly prescribed classes of oral hypoglycemic drugs, including metformin $[10,11]$. Both vildagliptin and fixed-dose combination vildagliptin/ metformin have been approved for therapeutic use in T2DM in the European Union and in various other countries.

In February 2008, a mandatory observational study monitoring the efficacy, tolerability, and safety profile of novel antidiabetic drugs for T2DM, including vildagliptin, in clinical practice for at least 1 year was started by the Italian Medicines Agency (Agenzia Italiana del Farmaco [AIFA]) [12]. This study derived from an addendum to the AIFA protocol and was designed to assess the hypothesis that fixed-combination vildagliptin/metformin improves work productivity measured as Work Productivity and Activity Impairment (WPAI) scores in the cohort of patients treated with the fixed combination after 1 year of treatment. Secondary objectives were the assessment of patient satisfaction by means of the Diabetes 
Treatment Satisfaction Questionnaire (DTSQs), the change in anthropometric measurements and in glucose control (HbA1c) after 1 year of treatment, and the evaluation of resource utilization (Resources Utilization Questionnaire).

\section{MATERIALS AND METHODS}

The study was a prospective, observational, multicenter study. Inclusion criteria were the following patient characteristics: adults with a diagnosis of T2DM, who were no longer responding to metformin monotherapy and to whom National Health Service (NHS) physicians prescribed fixed-combination vildagliptin/ metformin as a second step according to AIFA Registry on innovative drugs (incretin mimetics and DPP-4 inhibitors). This occurred during the monitoring period set by AIFA, in compliance with the recommendations described in the Summary of Product Characteristics [13]. The exclusion criteria were as follows: patients who were not able to complete the questionnaire independently, who had been taking any DPP-4 inhibitors for more than 1 month, and who required three or more antidiabetic drugs, or were using insulin.

Forty-nine NHS diabetes centers in Italy took part in the study, which was conducted in compliance with the decree on observational studies of March 2008 issued by AIFA and other relevant legislation. Patients had to give their informed consent to collection and use of the data in writing. The study was approved by local ethics committees.

The data collection included the following: demographic information (age, gender, ethnic group), anthropometric details (body weight, height, waist circumference, and calculation of body mass index [BMI]), medical history (date of diagnosis of T2DM, concomitant diseases), loss of productivity (WPAI scores), efficacy data (HbA1c), patient satisfaction data (DTSQ status scores), resource utilization (Resources Utilization Questionnaire outcome), exposure to the fixed combination, and study completion status.

The WPAI [14] is a six-item questionnaire aimed at evaluating the impact of a disease on absenteeism rate and work productivity over the last 7 days; it is completed by the patient. Four scores were derived from it: absenteeism (\% work time missed due to ill health, calculated as Q2/[Q2+Q4]), activity impairment (\% activity impairment due to ill health, calculated as Q6/10), work productivity score (\% overall work impairment due to health, calculated as [Q2+Q4×Q5/10]/ [Q2+Q4]), presenteeism (\% impairment while working due to health, calculated as Q5/10). However, considering the main study endpoint, presenteeism was not evaluated among the primary nor secondary variables, due to its lower impact on the NHS. For all the above scores, higher numbers indicate greater impairment and less productivity.

For the WPAI questionnaire, WPAI General Health (WPAI-GH) domains calculation, the following statements were adopted: if a patient declared not to be employed $(\mathrm{Q} 1=0)$, the remaining items dealing with work aspects (i.e., Q2, Q3, Q4, and Q5) were considered as missing values. If a patient declared not to be employed $(\mathrm{Q} 1=0)$ but even one item concerning work aspects (Q2, Q3, Q4, Q5) was compiled with a positive value $>0$, the patient was assumed to be employed (i.e., the Q1 answer was assumed to be 1). If a patient declared to be not employed or had Q1 missing value, and both Q2 and Q4 were = 0, the patient was assumed not to be employed (i.e., the Q1 answer was assumed to be 0 ). If a patient declared to be employed, the following consistency rules among work-answers were checked: 
- If Q4 (number of working hours during the last 7 days) $=0$, the value of the Q5 item (patient's productivity affected while working) was assumed to be missing.

- If Q2 (number of hours missed because of ill health), Q3 (number of hours missed because of other reasons), and Q4 (number of hours worked) items values were missing or $=0$, all the items Q2, Q3, Q4, and Q5 were assumed to be missing, although the patient maintained his/her employed status. If $\mathrm{Q} 2$ and $\mathrm{Q} 4=0$ but Q3 > 0, all the values were, instead, considered as possibly representing the condition of an employed patient absent from his/her workplace for reasons other than ill health (e.g., on holiday).

- If Q2 (number of hours missed because of health problem) or Q3 (number of hours missed because of other reasons) were missing but the Q4 (number of hours worked) value was a positive number $>0$, the Q2 and Q3 answers were assumed to be 0 (i.e., it was assumed that the patient had been working for the last 7 days).

For further details on each WPAI-GH question, see the Reilly Associated website [15].

DTSQ status [16] is an eight-item questionnaire, scored on a scale of $0-6$, with the aim of assessing total diabetes treatment satisfaction and the perceived frequency of hyperglycemia and hypoglycemia (5-6 = very dissatisfied; 3-4 = dissatisfied; $1-2$ = fairly satisfied; $0=$ very satisfied); it is completed by the patient. The Resource Utilization Questionnaire was completed by the physician in cooperation with the patient with the aim of assessing healthcare services utilization over the last 6 months and was subdivided into three areas: diabetic complications (cardiovascular, cerebrovascular, renal, neurologic, and ophthalmic), laboratory tests and other diagnostic investigations, other resources used (emergency department, general practitioner [GP] consultations, specialist consultations, hospitalizations). In order to assign a monetary value to these services, the official national tariff list was used [17-19]. The annual number of hours lost from work due to the disease was calculated based on the results of the questionnaire filled in at baseline and at the end of follow-up to the 1 -year period. For nonworking patients, it was assumed in a conservative manner that the same proportion of patients would have lost the same average number of hours from their regular weekly activities due to the disease as the working population. It was assumed that the calculated annual number of hours lost from work at baseline was representative of the year before, whereas the result obtained at the 12-month visit was representative of the 1 year of follow-up. In order to evaluate the annual cost of productivity lost due to the disease, the average annual number of hours lost from work by working patients was multiplied by the national average gross wage [20] per hour. The results were expressed as annual cost per working patient and annual cost per patient in general.

The patients were monitored for 1 year at baseline, 6 months, and at 12 months or at premature discontinuation. DTSQ and WPAI data were collected at baseline, 6 months and 12 months; the anthropometric and efficacy data at baseline and at 12 months; resource utilization at 6 and 12 months, referring to the previous 6 months. No baseline evaluation was available. The data other than DTSQ, WPAI, and resource utilization were already included in the AIFA monitoring project.

The data were collected on case report forms and the information was entered into 
an electronic database by means of single data entry with electronic data verification. Textual elements (e.g., comments) were verified manually. The entered data were checked by means of validation programs and listing control. Obvious errors were corrected by data management personnel, whereas nonobvious errors and omissions that generated queries were sent to the investigators for resolution. Concomitant diseases were coded using the terminology of the Medical Dictionary for Regulatory Activities (MedDRA). Values for continuous measures for patients who discontinued prematurely or had missing values were handled with the last observation carryforward (LOCF) approach for analysis. When the database was declared to be completed and accurate, it was locked.

\section{Statistical Analysis}

The statistical analysis was performed on all the patients treated with fixed-combination vildagliptin/metformin who had at least one post-baseline observation by Opis Srl (Desio, Italy), a clinical research organization, using SAS v. 9.1 (SAS Institute Inc., Cary, NC, USA). Demographic, anthropometric, efficacy, and resource utilization variables, as well as exposure, were analyzed descriptively, providing summary statistics for continuous variables and frequency tables for discrete variables. The mean changes in each calculated WPAI domain (higher numbers indicating greater impairment and less productivity) and in DTSQ satisfaction score (higher numbers indicating greater satisfaction) were analyzed by means of a paired $t$-test or signed-rank test (for non-normal data distributions). In addition, WPAI and DTSQ patients' rates for each score level were provided.

In order to reduce the impact of missing values on study results, the main efficacy analyses were performed both according to prevalence approach and according to LOCF approach. A sensitivity analysis was performed on those evaluable patients with both baseline and 12 month visit information with descriptive meaning.

The sample size was calculated on the basis of WPAI, which included three measures, so the Bonferroni adjustment method [21] was applied to safeguard the overall statistical significance level. Setting the alpha level at 5\%, power at $80 \%$, and assuming that the mean change after 52 weeks would range from -3.0 to -2.2 with a standard deviation between 21.7 and 23.0 on the basis of previous experience, a sample size of about 1,000 patients was required. With a sample size of 1,000 patients, the power was $97 \%$ for absenteeism, $83 \%$ for work productivity, and $80 \%$ for activity impairment.

\section{RESULTS}

A total of 1,046 patients were monitored and the majority of them (906 [86.6\%]), completed the study. Sixty-one of the 140 patients who did not complete the 12 months of observation had valid post-baseline data, which were carried forward up to the 12th month. Hence, 967 patients were included in the analysis. Out of the 140 patients who did not complete the study, 57 (5.5\%) withdrew their informed consent and 83 (7.9\%) were lost to follow-up. Mean \pm SD exposure to the fixed combination was $11.7 \pm 1.1$ months.

The patients were mostly Caucasian (98.6\%); nearly all the remaining patients were Asian (1.1\%). The population included patients of all ages, ranging from 28 to 88 years of age, but most of them were middle-aged (46-65 years; $62.1 \%$ ) or elderly ( $>65$ years; $30.6 \%$ ); $60.9 \%$ were of working age, but only $36.6 \%$ were actually working. Slightly more patients were male $(57.7 \%)$. 
On average, they had been diagnosed with T2DM $7.7 \pm$ standard deviation (SD) 6.7 years earlier; most of the patients had been diagnosed no more than 10 years earlier $(74.4 \%)$. More than $50 \%$ of the patients $(52.8 \%)$ had concomitant diseases: the most common were vascular disorders $(72.3 \%$; mainly hypertension $70.8 \%$ ), metabolism and nutrition disorders $(42.8 \%$, mainly dyslipidemia $19.2 \%$, hypercholesterolemia $12.1 \%$, and obesity $10.7 \%)$, and heart disease $(12.7 \%$, mainly ischemic heart disease 6.3\%).

All the three WPAI domains improved significantly after 1 year of treatment. Mean activity impairment improved significantly by $40.6 \%(P<0.0001)$. At baseline a total of 367 patients were actually working (36.6\%), and could provide absenteeism and total work productivity data; after 1 year absenteeism improved significantly by $49.9 \%(P=0.0076$, $n=248)$ and total work productivity score

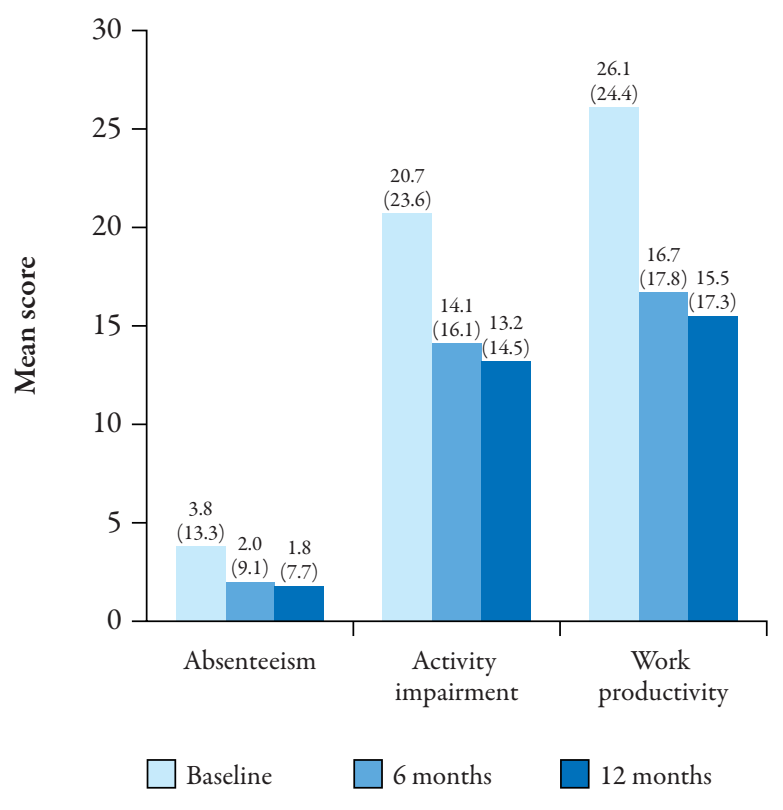

Fig. 1 Work Productivity and Activity Impairment Questionnaire: General Health (WPAI-GH): bar chart of scores by visit, according to prevalence approach (evaluable patients) improved significantly by $37.6 \%(P<0.0001$, $n=244)$. Major improvement was already recorded after 6 months of treatment (Fig. 1). The LOCF approach yielded similar results. The average number of hours per week reported to be lost due to diabetes decreased by $46 \%$ at the end of follow-up (1.64 vs. 0.63 ). At baseline, $11.7 \%$ of working patients reported to have lost due to the disease on average 11.4 hours in the last week. At the end of follow-up $9.8 \%$ of working patients reported to have lost on average 6.1 hours in the last week due to the disease. The number of hours lost per patient due to illness during the previous year was 59 , with a reduction of $56 \%$ in the year of follow up, resulting in 26 hours lost per patient (Table 1). In monetary terms, considering only the wage of working patients, there was a 55\% reduction equivalent to $€ 400.15$ (€721.13 vs. $€ 321.98$ ) on average per employed patient. Considering all patients (both working and nonworking) the average reduction was 53\% equivalent to $€ 134.85$ ( $€ 253.1$ vs. $€ 118.17$ ) per patient (Table 2).

The mean (SD) TTS score increased significantly by $30.2 \%$ ( $29.6 \pm 5.6$ vs. $22.8 \pm 6.9$; $P<0.0001)$ after 1 year. The proportion of patients who were very satisfied with treatment increased from baseline by $44.7 \%$, those who perceived themselves as hyperglycemia-free or almost hyperglycemia-free by $37.9 \%$, and those who perceived themselves as hypoglycemia-free or almost hypoglycemia-free by $15.2 \%$ (Fig. 2).

A post-hoc analysis comparing activity impairment status (worsened, i.e., change vs. baseline $>0$; stable, i.e., change vs. baseline $=0$; improved, i.e., change vs. baseline $<0$ ) with the perception of hypoglycemia (worsened, stable, improved) showed that there was a statistically significant association between stable and improved activity impairment and stable or improved perception of hypoglycemia (chi-square $P<0.0001)$. Activity impairment and perception 
Table 1 Indirect costs: h of productivity lost

\begin{tabular}{|c|c|c|c|}
\hline & \multicolumn{2}{|c|}{ Total number of patients } & \multirow{3}{*}{ Difference } \\
\hline & Baseline & Month 12 & \\
\hline & $1,046(100 \%)$ & $967(100 \%)$ & \\
\hline \multicolumn{4}{|l|}{ Working patients } \\
\hline Total number of working patients & $367(35 \%)$ & $356(37 \%)$ & \\
\hline Number of working patients with no productivity loss & 256 & 253 & \\
\hline Number of working patients with missing data & 68 & 68 & \\
\hline Number of working patients with productivity loss (\% of & $43(11.7 \%)$ & $35(9.8 \%)$ & \\
\hline working patients) & 11.4 & 6.1 & -5.3 \\
\hline \multicolumn{4}{|l|}{ Average number of $h$ in a week missed by working patients } \\
\hline \multicolumn{4}{|l|}{ Nonworking patients } \\
\hline Total number of nonworking patients & $679(65 \%)$ & $611(63 \%)$ & \\
\hline Number of nonworking patients that might have lost h due to disease & 80 & 60 & \\
\hline Average number of $\mathrm{h}$ in a week missed by nonworking patients & 11.4 & 6.1 & -5.3 \\
\hline Total number of patients with missed h (working and nonworking) & 123 & 95 & \\
\hline Total number of missed $\mathrm{h}$ by working and nonworking patients in a week & 1,399 & 576 & -823 \\
\hline Total number of missed h by working and nonworking patients in a year & 61,575 & 25,365 & $-36,210$ \\
\hline Number of h per patient per year lost due to the illness & 59 & 26 & $-33(-56 \%)$ \\
\hline Number of h missed per patient with missed h per year & 501 & 206 & $-295(-59 \%)$ \\
\hline
\end{tabular}

After 1 year of treatment the annual number of h per patient missed for the disease management decreased by $56 \%$ $11.7 \%$ of patients $(n=123)$ lost on average $501 \mathrm{~h}$ in the year before and $206 \mathrm{~h}$ during the 1 -year follow-up

Table 2 Indirect costs: the cost of productivity loss

\begin{tabular}{|c|c|c|c|}
\hline & Baseline & Month 12 & Difference (\%) \\
\hline Total cost of productivity lost & $€ 264,652.96$ & $€ 114,268.00$ & $-€ 150,384.96$ \\
\hline Number of employed patients & $n=367$ & $n=356$ & \\
\hline Cost per employed patient per year & $€ 721.13$ & $€ 320.98$ & $-€ 400.15(-55)$ \\
\hline Number of patients (all) & $n=1,046$ & $n=967$ & \\
\hline Cost per patient per year & $€ 253.01$ & $€ 118.17$ & $-€ 134.85(-53)$ \\
\hline
\end{tabular}

Indirect cost per patient per year has decreased by $53 \%$ at the end follow-up

Indirect cost per employed patient per year has decreased by $55 \%$ at the end follow-up

of change in hyperglycemia were both either stable or improved in $73 \%$ of patients.

The anthropometric measures improved during the 1 year of treatment in women, but remained almost the same in men: mean \pm SD waist circumference diminished by $-1.8 \mathrm{~cm}$ in women $(102.1 \pm 12.9 \mathrm{~cm}$ vs. $103.5 \pm 13.5 \mathrm{~cm}$ at baseline) and by $-0.6 \mathrm{~cm}$ in men $(103.7 \pm 11.5 \mathrm{~cm}$ vs. $104.5 \pm 11.3 \mathrm{~cm}$ at baseline); mean body weight diminished by $-4.2 \mathrm{~kg}$ in women $(77.8 \pm 15.5 \mathrm{~kg}$ vs. $79.3 \pm 16.2 \mathrm{~kg}$ ), whereas it increased by $+1.2 \mathrm{~kg}$ in men $(85.5 \pm 14.6 \mathrm{~kg}$ vs. $86.3 \pm 15.0 \mathrm{~kg}$ at baseline); also BMI diminished by $-1.3 \mathrm{~kg} / \mathrm{m}^{2}$ in women $(29.1 \pm 6.8$ vs. $31.6 \pm 6.2$ at baseline $)$ whereas it increased by $+0.7 \mathrm{~kg} / \mathrm{m}^{2}$ in men $(31.2 \pm$ 6.3 vs. $29.6 \pm 4.6$ at baseline). 
(a)

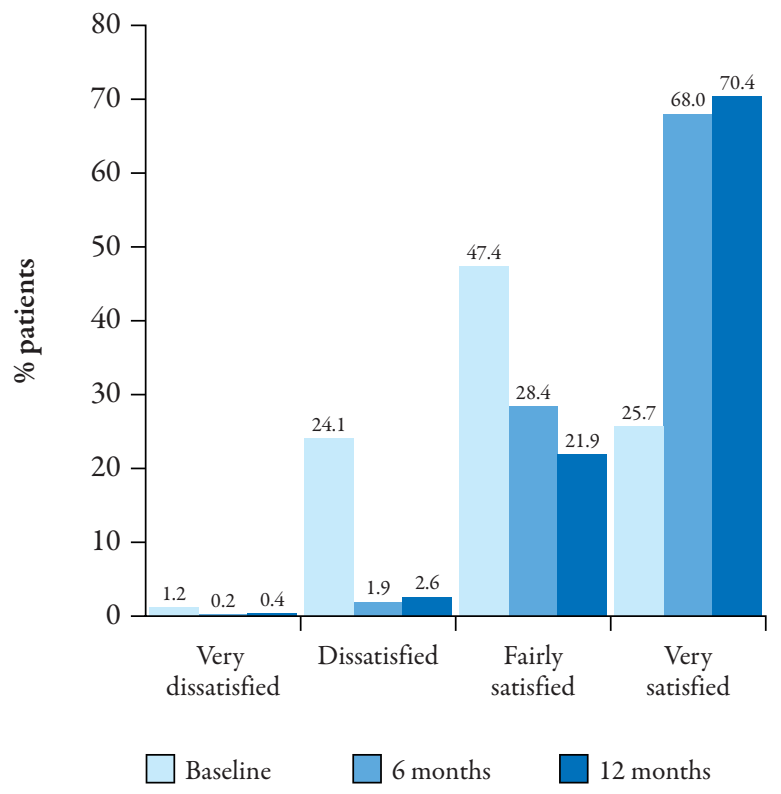

(c)

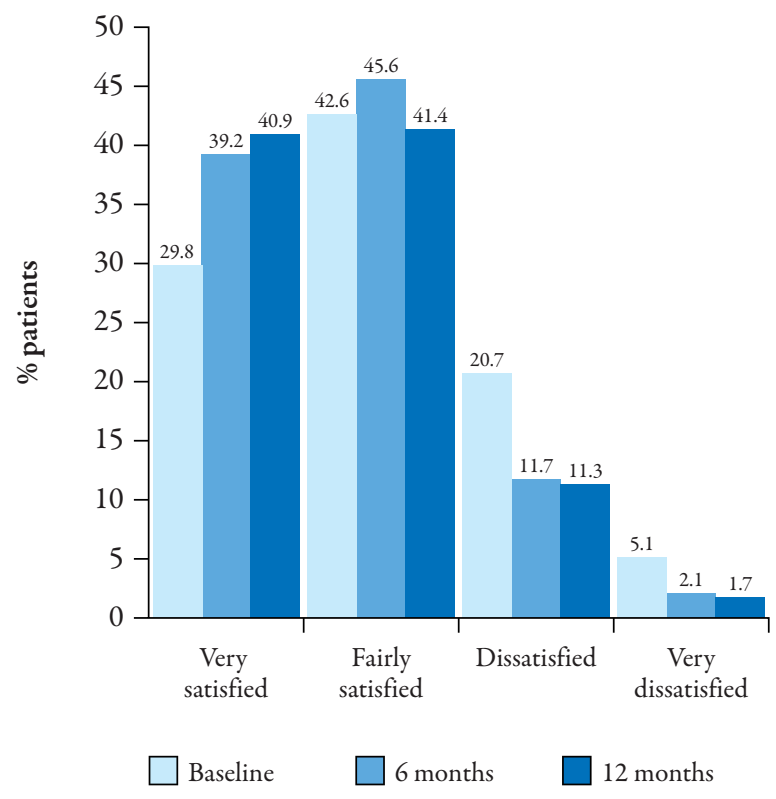

Fig. 2 (a) Proportion of patients by extent of satisfaction with current treatment expressed as a score on a sixitem semiquantitative rating scale. (b) Proportion of patients by perception of hyperglycemic episodes expressed as a score on a six-item semiquantitative rating scale (c) Proportion of patients by perception of hypoglycemic episodes expressed as a score on a six-item semiquantitative rating scale (b)

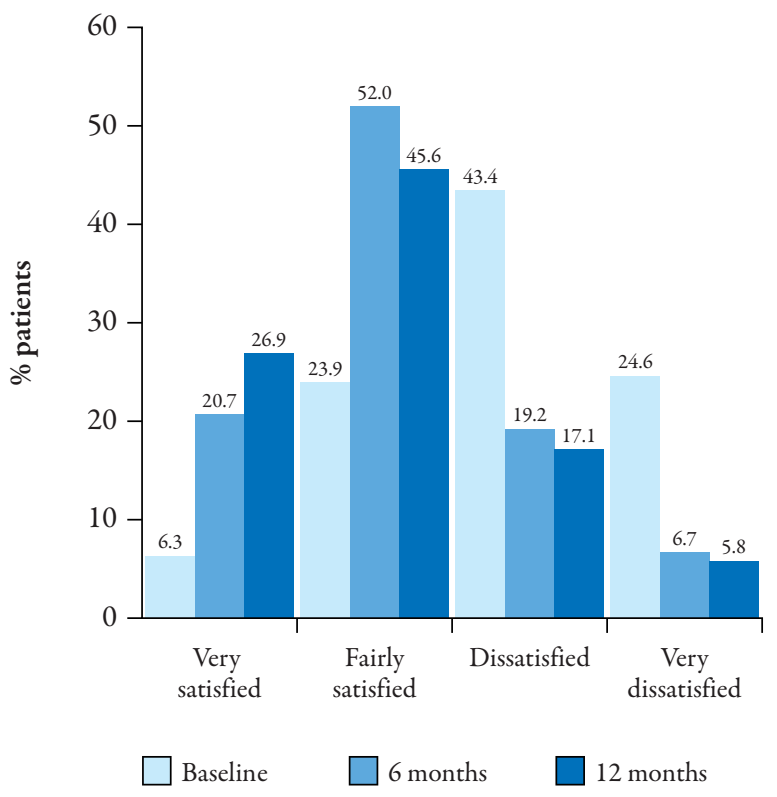

Efficacy expressed as HbA1c improved with the fixed combination in $54 \%$ of patients and remained stable in $41 \%$. Mean HbA1c diminished by $0.9 \%$ from $8.0 \pm 1.2 \%$ down to $7.1 \pm 0.9 \%$. There was no important difference between genders and BMI.

In total, 20 patients (2.2\%) were admitted to hospital: 16 because of complications and 4 because of unsatisfactory glucose control. Nearly all the patients (96.5\%) had laboratory and/or other diagnostic tests, on average 22.3 per patient during the 1 year follow-up. In particular, nearly all the patients were prescribed HbA1c (96.3\%; mean number 2.8), fasting blood sugar (91.6\%; mean number 8.9), and alanine aminotransferase/ aspartate aminotransferase (ALT/AST; 95.6\%; mean number 2.7). Most patients were prescribed high-density lipoprotein/low-density lipoprotein (HDL/LDL) cholesterol (87.8\%; mean number $2.2)$, triglycerides (87.8\%; mean number 2.2$)$, complete urinalysis (82.0\%; mean number 2.3). Other commonly prescribed tests were uric acid (60.7\%; mean number 1.8) and microalbuminuria (64.3\%; mean number 2.1). A total of 
804 patients (80.2\%) used additional resources, on average 6.2 times: $65.7 \%$ of patients consulted their GP on average 3.5 times, $60.5 \%$ consulted a specialist on average 2.8 times, an average of 1.5 additional investigations (instrumental exams/tests) were prescribed to $17.4 \%$ patients, and $2.6 \%$ of patients went to an emergency room on average 1.3 times.

The mean healthcare cost per patient diminished from $€ 130.22$ during the first semester to $€ 105.28$ in the second semester $(-19.2 \%)$; the annual cost per patient was $€ 225.33$. Resource breakdown showed that all the kinds of resources considered diminished, reductions ranging from $-15.3 \%$ (laboratory tests and investigational procedures) to $-23.2 \%$ (hospitalizations; Fig. 3).

\section{DISCUSSION}

Incretin mimetics are innovative drugs for oral treatment of T2DM and their recent introduction into clinical practice would be expected to have a negative impact on diabetic therapy costs.

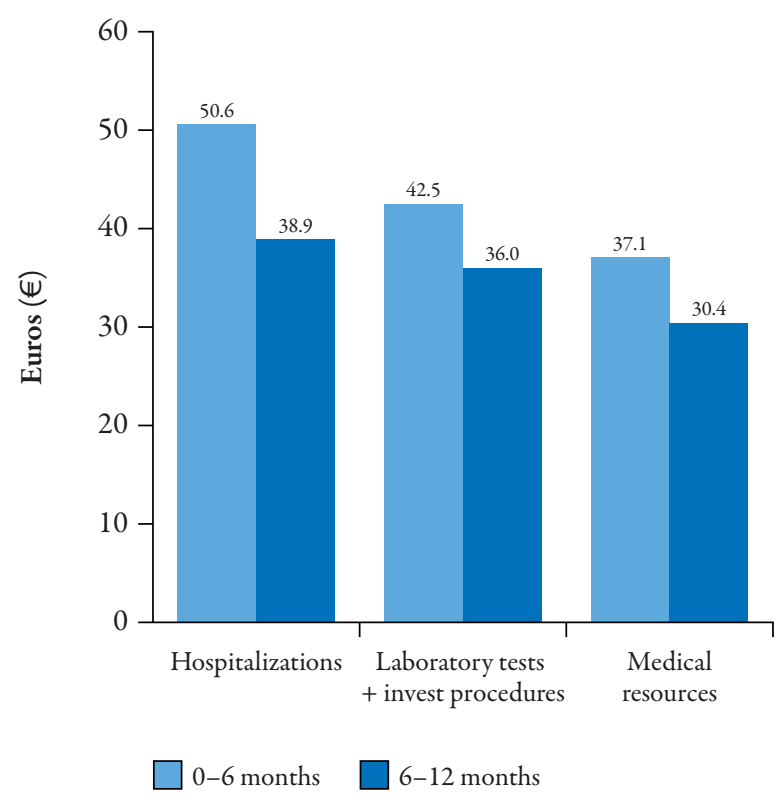

Fig. 3 Comparison of the mean costs per patient during the first and second semester of treatment in Euros
This study shows that the use of fixedcombination vildagliptin/metformin for 1 year is associated not only with a significant improvement in work productivity $(+40.6 \%)$, but also with an annual reduction in indirect costs of $€ 400.15$ per working patient and of $€ 134.85$ per patient in general. It also shows that the use of the fixed combination is associated with an improvement in quality of life, as measured by the DTSQ questionnaire $(+30.2 \%)$, particularly in terms of perception of excessive changes in blood glucose levels (hyperglycemia $+37.9 \%$, hypoglycemia $+15.2 \%$ ); these perceptions are supported by an objective improvement in HbA1c in $54 \%$ of patients and stabilization of HbA1c in $41 \%$. An estimate of healthcare costs per patient suggested that the use of fixedcombination vildagliptin/metformin actually reduces such costs, as they diminished on average by $19 \%$ during the second semester of treatment. The results related to productivity and quality of life applies to all countries. The estimates of costs may vary from country to country, but the outcome that healthcare expenditure tends to diminish should apply everywhere.

The results of the present study are consistent with the outcome of a survey in 1,404 respondents by the Brod group, who established that nonsevere hypoglycemic events are associated with lost productivity estimated to range from $\$ 15.26$ to $\$ 93.47$ per event and from 8.3 to 15.9 hours of lost work time per month [22].

To the authors' knowledge, no other studies have provided data on the impact of fixedcombination vildagliptin/metformin on work productivity and indirect cost saving using WPAI, or on patient satisfaction measured by DTSQ. Thus, the strength of the study is that it has provided additional original information that enables a more complete evaluation of the 
global impact of chronic antidiabetic treatment with fixed-combination vildagliptin/metformin on the life of the patient.

Extensive data have already been provided on the efficacy of the vildagliptin/metformin combination in reducing $\mathrm{HbA1c}$, which are consistent with the results in this study $(-0.6$ to $-1.8 \%$ vs. $-0.9 \%$ in the present study) [23-26]. The finding of improved satisfaction with treatment and improved $\mathrm{HbA} 1 \mathrm{c}$ is consistent with the outcome of a study on quality of life in 2,500 outpatients with T2DM, which found that improvement in HbA1c and the use of oral antidiabetic agents only were amongst the factors that contributed to better quality of life, particularly to higher treatment satisfaction as measured by DTSQ [27].

The assessment of utilization of healthcare resources other than drugs and the consequent cost for patients with T2DM treated with the fixed combination suggested that the introduction of the new antidiabetic product did not increase healthcare resource utilization and related costs; on the contrary it appeared to reduce it. The estimate of annual cost per patient of $€ 225.00$ is lower than the annual cost per patient (€320.00-€340.00) reported in an Italian study [28]. The reason for this difference may lie in differences in the patient population, as Garattini et al. included also patients on insulin, who may experience more complications. The assessment of direct healthcare costs is lower also than in previous observational studies [29]. As the study did not consider the cost of drugs, it is not fully consistent with a previous assessment of the economic impact of the introduction of vildagliptin, both alone and in fixed combination with metformin, on healthcare expenditure for T2DM patients in Italy [30]. This assessment ascertained that the introduction of vildagliptin involved an increase in pharmaceutical costs that was offset by a reduction in the management costs of serious or severe adverse events (which were fewer as specified below and documented in the Periodic Safety Update Reports on metformin, vildagliptin) [31] and in therapeutic monitoring. Nevertheless, the outcome was that introduction of the fixed combination produced an overall increase in healthcare expenditure by $1.48 \%$, a modest increase, but not no effect at all or even a reduction as this study suggests. Failure to consider the cost of drugs, which makes this study not fully consistent with previous investigations, is a limitation of this study.

Fewer adverse events are the result of the good tolerability profile of fixed-combination vildagliptin/metformin. Safety and tolerability were assessed according to the regulations in force and the recommendations in the summary of product characteristics within the context of the study conducted by AIFA on innovative antidiabetic drugs, of which this study is an addendum. The AIFA study was an important component of the usual mandatory pharmacovigilance plan for medicinal products that have been recently introduced into clinical practice. From January 2009 to July 2011 only 69 adverse reactions were reported in the whole patient population given the fixed combination ( $n=21,483$ patients). Of the 69 reactions, only $12(0.06 \%)$ were serious [31]. This is consistent with the outcome of this study, in which only 20/906 patients $(2.2 \%)$ were hospitalized because of serious adverse events. Consequently, the fixed combination is also well tolerated in reallife clinical practice; its safety and tolerability profile may be considered to be reliable.

The good tolerability profile of fixedcombination vildagliptin/metformin may explain the low assessment of costs: a survey in more than 20,000 adult patients with T2DM on treatment with oral antidiabetic agents and not 
insulin, as in this study, showed that there is an inverse association between productivity (as measured by WPAI) and tolerability [32].

The hospitalization rate was particularly low in this study (2.2\%), because it included only adult patients suffering from type 2 diabetes mellitus that was not severe and was well controlled by oral antidiabetic therapy. The study excluded patients on insulin, who experience the most complications and who consume the most resources. This is a weakness of the study and may account for the particularly low estimation of resource utilization costs, resource consumption, and related annual cost per patient.

\section{CONCLUSION}

This observational study suggests that the fixed combination of vildagliptin/metformin increases work productivity, reducing indirect costs, and improves quality of life, especially in terms of perception of changes in blood glucose, in patients with T2DM. This additional information should be taken into consideration for the overall evaluation of the clinical impact of the combination.

\section{ACKNOWLEDGMENTS}

Sponsorship and article processing charges for this study was funded by Novartis Farma S.p.A. Dr. Genovese designed the study, supervised the conduct of the study from a scientific point of view, interpreted the results, and critically reviewed the manuscript. Dr. Tedeschi coordinated the study team, contributed to interpreting the results, and drafted the manuscript. This study was funded by Novartis Farma S.p.A. Editorial assistance in the preparation of the manuscript was provided by Dr. Jennifer S. Hartwig. Support for this assistance was funded by Novartis Farma S.p.A. Dr. Genovese is the guarantor for this article, and takes responsibility for the integrity of the work as a whole.

The authors express gratitude towards the following.

The Investigator's Team. M. Dolci and F. Baccetti (Massa), A. Francesconi, and A. Ladu (Firenze), C. Baggiore (Firenze), A. Di Carlo (Lucca), R. Bruno (Pisticci, Matera), M. Barbagallo and L. J. Dominguez Rodriguez (Palermo), G. Sodo (Caserta), M. Rizzo (Palermo), M. Bonomo and O. Disoteo (Milano), T. De Franceschi and R. Durante (Albenga, SV), A. Mastropasqua and Marenco (Garbagnate Milanese, MI), A. Luciano (Benevento), M. Trovati and A. Cavallo (Orbassano, TO), M. L. Fatti (Milano), F. Privitera (Catania), B. Tizio (Eboli, SA), P. Calatola and Loconte (Salerno), E. Orsi and E. Palmieri (Milano), GMM. Grasso (Catania), G. Reina (Biancavilla, CT), G. Allochis and Aimaretti (Novara), AV. Ciardullo (Carpi, MO), G. Grossi and R. Piro (Paola, CS), L. Tramontano and G. De Morelli (Praia a Mare, CS), G. Maolo and B. Polenta (Macerata), L. Valente (Frosinone), F. Travaglino (Biella), G. Carlesi and M. Lanero (Novi Ligure, AL), M. Carlini and A. Blatto (Torino), C. Tubili and L. Morviducci (Roma), D. Caputo and L. Landolfi, Oliva (Salerno), R. Bulzomì (Roma), N. Padovano and D. Caggiano (Salerno), E. Petraroli (Monte di Procida, NA), G. Bargero (Casale Monferrato, AL), V. Iurlaro (Francavilla Fontana, BR), A. Sasso (Trani, BA), C. M. Rotella and E. Romoli (Firenze), R. Manfredini and M. L. Grata (Lagosanto, FE), A. Fabbri and M. Giuliano (Roma), M. A. Pellegrini (Udine), C. Giovannini (Reggio Calabria), A. M. Aiello (Martina Franca, TA), V. Provenzano and Ferrara, G. Saura (Partinico, PA), G. Fatati and I. Grandone, E. Mirri (Terni), E. Forte (Terracina, LT) for the recruitment and management of participating patients. 
The Sponsor's Team. Marta Bartezaghi and Elena Peruzzi from Epidemiology and Outcome Research, and Federico Bertocchi Cardiovascular and Metabolic Therapeutic Area, Medical Department, at Novartis Farma S.p.A, for information about the study drug and use of rating scales.

Conflict of interest. Dr. Genovese received speaking fees and consultancy honoraria from Novartis Farma S.p.A. Dr. Tedeschi is working in the Medical Department of Novartis Farma S.p.A.

Open Access. This article is distributed under the terms of the Creative Commons Attribution Noncommercial License which permits any noncommercial use, distribution, and reproduction in any medium, provided the original author(s) and source are credited.

\section{REFERENCES}

1. DM News (Medical Department Bulletin) - December 2012. Data on file. Italy; Novartis Farma S.p.A.; 2010.

2. Jönsson J. Revealing the cost of type II diabetes in Europe. Diabetologia. 2002;45:S5-12.

3. Diabetes fact sheet. Available at: www.who.int/ mediacentre/factsheets/fs312/en/index.html. Accessed Aug 262011.

4. National Center for Chronic Disease Prevention and Health Promotion. Fact sheet. Diabetesatwork. org. Available at: http://www.cdc.gov/diabetes/ pubs/factsheets/atwork.htm. Accessed Jan 152013.

5. Holmes J, Gear E, Bottomley J, Gillam S, Murphy M, Williams R. Do people with type 2 diabetes and their carers lose income? (T2ARDIS-4) Health Policy. 2003;64:291-6.

6. Lucioni C, Garancini MP, Massi-Benedetti M, Mazzi S, Serra G, The costs of type 2 diabetes mellitus in italy a CODE-2 sub-study. Treat Endocrinol. 2003;2:121-33.

7. International Diabetes Federation Clinical Guidelines Task Force. Global Guideline for type 2 diabetes. 2005. Available at: http://www.staff.ncl. ac.uk/philip.home/IDF\%20GGT2D.pdf. Accessed Jan 152013.
8. UKPDS Study Group. Overview of six years' therapy of type 2 diabetes, a progressive disease. Diabetes. 1995;44:1249-58.

9. Turner RC, Cull CA, Frighi V, Holman RR. Glycemic control with diet, sulfonylurea, metformin or insulin in patients with type 2 diabetes mellitus: progressive requirement multiple therapies (UKPDS 49). UK Prospective Diabetes Study (UKPDS) Group. JAMA. 1999;281:2005-12.

10. Dejager S, Baron MA, Schweizer A. Emerging treatments for type 2 diabetes. DPP- 4 inhibitors: review of vildagliptin phase 3 data. Rev Endocrinol. 2007;1:47-51.

11. Ahren B, Foley JE, Bosi E. Clinical evidence and mechanistic basis for vildagliptin's action when added to metformin. Diabetes Obes Metab. 2011;13:193-203.

12. Registro farmaci antidiabetici sottoposti a monitoraggio - Rapporto farmaci incretino-mimetici e DPP-4 inibitori. Available at: http://antidiabetici. agenziafarmaco.it/ [In Italian]. Accessed Jan 152013.

13. Eucreas $50 \mathrm{mg} / 850 \mathrm{mg}$ and $50 \mathrm{mg} / 1000 \mathrm{mg}$ filmcoated tablets. Summary of Product Characteristics. Last updated on the eMC: 09/01/2013. Available at: www.medicines.org.uk/emc/medicine/20735/ SPC/. Accessed Jan 172013.

14. Reilly MC, Zbrozek AS, Dukes EM. The validity and reproducibility of work productivity and activity impairment instrument. PharmacoEconomics. 1993;4:353-65.

15. Reilly Associates. WPAI Scoring. Available at: http:// www.reillyassociates.net/WPAI_Scoring.html. Accessed Jan 152013.

16. Bradley C. The Diabetes Treatment Satisfaction Questionnaire: DTSQ. In: Bradley C, (ed). Handbook of Psychology and Diabetes: a Guide to Psychological Measurement in Diabetes Research and Practice. Chur: Harwood, 1994:111-32.

17. Hospital care service tariffs for acute cases hospitalized in a ward or in a day hospital unit [in Italian]. Ordinary Supplement of Official Gazette. no 289, 2006.

18. Specialist care services in clinics disbursed by the National Health Service and relative tariffs. [In Italian]. Available at: http://www.trovanorme.salute. gov.it/dettaglioAtto?id=6403. Accessed Jan 302013.

19. Lucioni C, Mazzi S, Serra G. [L'impatto delle complicanze diabetiche sui costi sanitari e la QoL del paziente con Diabete di Tipo 2: i risultati dello studio CODE-2]. Il Diabete. 2000:275-86. In Italian. 
20. Banca d'Italia - Relazione Annuale sul 2009, 31 maggio 2010. Available at: http://www. bancaditalia.it/pubblicazioni/relann/rel09 [In Italian]. Accessed Jan 172013.

21. Bland JM, Altman DG. Multiple significance tests: the Bonferroni method. BMJ. 1995;310:170

22. Brod M, Christensen T, Thomsen TL, Bushnell DM. The impact of non-severe hypoglycemic events on work productivity and diabetes management. Value Health. 2011;14:665-71.

23. Bosi E, Rochotte E, Camisasca RP, Garber AJ, Collober C. Effects of vildagliptin on glucose control over 24 weeks in patients with type 2 diabetes inadequately controlled with metformin. Diabetes Care. 2007;30:890-5.

24. Bolli G, Dotta F, Colin L, Minic B, Goodman M. Comparison of vildagliptin and pioglitazone in patients with type 2 diabetes inadequately controlled with metformin. Diabetes Obes Metab. 2009;11:589-95.

25. Bosi E, Dotta F, Jia Y, Goodman M. Vildagliptin plus metformin combination therapy provides superior glycaemic control to individual monotherapy in treatment-naive patients with type 2 diabetes mellitus. Diabetes Obes Metab. 2009;11:506-15.

26. Schweizer A, Dejager S, Foley JE, Shao Q, Kothny W. Clinical experience with vildagliptin in the management of type 2 diabetes in a patient population $\geq 75$ years: a pooled analysis from a database of clinical trials. Diabetes Obes Metab. 2011;13:55-64.
27. Nicolucci A, Cucinotta D, Squatrito $\mathrm{S}$ et al. Clinical and socio-economic correlates of quality of life and treatment satisfaction in patients with type 2 diabetes. Nutr Metab Cardiovasc Dis. 2009;19:45-53.

28. Garattini L, Chiaffarino F, Cornago D, Coscelli C, Parazzini F, Direct medical costs unequivocally related to diabetes in Italian specialized centers, Eur J Health Econom. 2004;5:15-21.

29. Marchesini G, Forlani G, Rossi E, Berti A, De Rosa $\mathrm{M}$ on behalf of the ARNO Working Group. The direct economic cost of pharmacologically-treated diabetes in Italy - 2006. Nutr Metab Cardiovasc Dis. 2011;21:339-46.

30. Zaniolo O, Sacchi V, Pradelli L. [Impatto sul budget del SSN dell'impiego di vildagliptin, un nuovo ipoglicemizzante orale.] Farmacon Percorsi Terap. 2008;9:137-46. In Italian.

31. Periodic Safety Update Report 4 (PSUR 4). Period covered: 01 Jan 2009 - 30 Nov 2009; Periodic Safety Update Report 5 (PSUR 5). Period covered: 01 Dec 2009 - 30 Nov 2010; PSUR Addendum Report 01. Period covered: 01 Dec 2010-20 Jul 2011. Data on file. Italy; Novartis Farma S.p.A.; 2011.

32. Di Bonaventura M, Link C, Pollack MF, Wagner JS, Williams SA. The relationship between patientreported tolerability issues with oral antidiabetic agents and work productivity among patients having type 2 diabetes. J Occup Environ Med. 2011;53:204-10. 